\title{
Clinical aggressiveness of TP53-wild type Sonic Hedgehog medulloblastoma with $M Y C N$ amplification, chromosome 17p loss, and chromothripsis
}

\author{
Yuichi Mitani $^{1}$, Kohei Fukuoka ${ }^{1}$, Makiko Mori ${ }^{1}$, Yuki Arakawa ${ }^{1}$, Yuko Matsushita ${ }^{2}$, Yuko \\ Hibiya $^{2}$, Satoko Honda ${ }^{1}$, Masao Kobayashi ${ }^{1}$, Yutaka Tanami ${ }^{1}$, Yonehiro Kanemura ${ }^{3}$, \\ Atsuko Nakazawa ${ }^{4}$, Jun Kurihara ${ }^{1}$, Katsuyoshi Koh ${ }^{1}$, and Koichi Ichimura ${ }^{2}$ \\ ${ }^{1}$ Saitama Children's Medical Center \\ ${ }^{2}$ National Cancer Center Research Institute \\ ${ }^{3}$ National Hospital Organization Osaka National Hospital \\ ${ }^{4}$ Saitama Kenritsu Shoni Iryo Center
}

July 16, 2020

\begin{abstract}
A recently proposed risk stratification of medulloblastomas has shown that Sonic Hedgehog (SHH) subtype with TP53 mutation is the worst prognostic. Here, we describe the case of a 6-year-old boy with clinically very aggressive SHH medulloblastoma like TP53 mutant, but the genetic status of the case was wild type. Copy number analysis showed $M Y C N$ amplification, chromosome 17p loss, and chromothripsis, which are known to be strongly associated with TP53-mutated SHH tumors. The presence of both chromosome 17p loss and chromothripsis in SHH medulloblastoma may suggest a p53 pathway dysregulation regardless of the TP53 status, leading to a much worse prognosis.
\end{abstract}

\section{Introduction}

Owing to research advances, medulloblastomas can be divided into four molecular subtypes: WNT, SHH, group 3 , and group $4^{1,2}$. In the most recent World Health Organization classification of Tumors of the Central Nervous System ${ }^{3}$, the molecular subclassification was employed as WNT-activated, SHH-activated, and NonWNT/Non-SHH. Based on molecular subgrouping in combination with a number of genetic alterations, a renewed risk stratification of the tumors has recently been proposed to improve patient survival and reduce late effects ${ }^{1}$.

TP53 status is critical for SHH medulloblastomas since the presence of the mutation is regarded as one of the poorest prognostic factors for this subtype ${ }^{4,5}$. In the aforementioned updated risk stratification, TP53 -mutant SHH has been designated as a "very high risk" group ${ }^{1}$.

Here, we report clinically very aggressive SHH medulloblastoma like TP53 mutant tumors but the genetic status of the case was wild type. The case has peculiar copy number abnormalities of $M Y C N$ amplification, chromosome (chr) 17p loss, and chromothripsis, which were reported to have a strong correlation with p53 pathway dysregulation in SHH tumors ${ }^{6}$.

\section{Case Description}

A 6-year old boy with no medical history presented with frequent vomiting. Enhanced magnetic resonance imaging (MRI) of the brain revealed a mass lesion sized $50 \times 41 \times 38 \mathrm{~mm}$, located in the upper middle cerebellum (Figs. 1A, B). Neither spinal MRI nor cytology of cerebrospinal fluid suggested spinal dissemination 
of the tumor. The patient underwent insertion of an external ventricular drain followed by craniotomy for tumor removal, and gross-total resection was achieved. The pathology examination revealed small round cells with marked nuclear anaplasia, prominent nucleoli, as well as "cell wrapping" appearance, which resulted in the diagnosis of large cell/anaplastic (LC/A) medulloblastoma (Fig. 1D). The patient received 4 courses of chemotherapy (vincristine, cisplatin, etoposide, cyclophosphamide, and intrathecal methotrexate), 24.0 Grey (Gy) of craniospinal irradiation, and 51.2 Gy of local radiation therapy. We eventually decided to add high-dose chemotherapy composed of thiotepa and melphalan to augment treatment because the tumor was molecularly diagnosed as SHH with MYCN amplification by Nanostring and interphase fluorescencein situ hybridization (iFISH) (Fig. 1E) after treatment initiation. Unexpectedly, the p53 immunohistochemistry (IHC) was negative (Fig. $1 \mathrm{~F}$ ) and the TP53 mutation analysis from exons 2 to 11 showed wild type in this case.

Complete remission was achieved at the end of treatment. However, the patient soon presented with anorexia and leg pain, and MRI showed massive neuroaxis dissemination (Fig. 1C). Despite administering 36 Gy of additional spinal irradiation concomitant with temozolomide administration, the patient died with progressive disease ten months after the initial diagnosis.

Afterwards, the methylation data of the case was entered into a recently published classifier ${ }^{7}$, which classified the tumor as methylation class "medulloblastoma, subclass SHH A (children and adult)" with high confidence (calibrated score was 0.93). Copy number analysis showed $M Y C N$ amplification, which was consistent with iFISH results, as well as loss of chr 17p where TP53 is located (Fig. 2). There was also a peculiar chromosomal abnormality, which seemed to be chromothripsis in chr 13.

\section{Discussion}

SHH medulloblastomas are a distinct molecular medulloblastoma subgroup, but they have substantial biological heterogeneity ${ }^{1,2,8}$. Cavalli et al. reported that the SHH subgroup could be further subdivided into 4 groups called $\alpha, \beta, \gamma$, and $\delta$. Our case may be included in SHH $\alpha$ due to the similarity of the clinical features and copy number abnormalities (MYCNamplification, chr $17 \mathrm{p}$ loss $)^{8}$.

In SHH cases, the presence of a TP53 mutation indicates a dismal prognosis. Zhukova et al. revealed that the TP53 mutation was a subgroup-specific prognostic factor in not WNT but SHH group and most of the patients with TP53 -mutant SHH medulloblastoma succumbed in 2 years from the diagnosis, which was very similar to the clinical course of our patient ${ }^{4}$. The case showed wild type of the gene by both mutation analysis and IHC, with extensive mutation analysis covering the coding region of the gene ${ }^{9}$, reported as mutation hotspots among the subtype of medulloblastomas in a previous report ${ }^{4}$.

Because of the presence of chromothripsis, the p53 pathway disruption may have occurred without the truncated genetic mutation in our case. Chromothripsis is a massive catastrophic genomic event, which may include chromosome shuttering followed by random structural rearrangement, leading to the development of cancer ${ }^{6}$. Rausch et al. found that chromothripsis mostly develops in the SHH subgroup with TP53 mutation, in contrast to very few occurrences in other medulloblastomas ${ }^{6}$. TP53 mutation as an early event in tumor development can facilitate chromothripsis in cells by a couple of possible effects of the mutated gene, which includes telomere attrition and following chromosomal end-to-end fusion development ${ }^{6}$. As an exception, several wild-type TP53 SHH medulloblastoma cases actually presented chromothripsis as well as TP53 -mutant tumors, and all of them showed chr $17 \mathrm{p}$ loss, similar to our case ${ }^{6}$. Clinically, a large international collaborative cohort by Shih et al. revealed that patients with SHH medulloblastomas showing chromothripsis had significantly worse clinical outcomes than those without the copy number abnormality ${ }^{10}$.

Chr $17 \mathrm{p}$ loss itself could be involved in the development of cancer through another process. Liu et al. demonstrated the fact that, in mice, heterozygous deletion of chr $17 \mathrm{p}$ has a higher effect to drive tumorigenesis of leukemia/lymphoma than the TP53 deletion alone ${ }^{11}$. They also demonstrated that specific genes located close to TP53 on the chromosome 17 would make tumors more aggressive due to the combined effect of TP53loss ${ }^{11}$. 
Amplification of $M Y C N$ may also have a great effect on the aggressiveness of this case. Kool et al. and Shwarlbe et al. showed that $M Y C N$ amplification was an independent poor prognostic factor in the SHH medulloblastoma subgroup ${ }^{12,13}$. Whereas, Korshunov et al. demonstrated that $M Y C N$ amplification was associated with a favorable outcome in terms of patient survival ${ }^{14}$. The controversy might be explained by the frequent co-occurrence of TP53 mutations in these subgroups ${ }^{4,14}$.

In conclusion, the clinical and molecular integrated analysis of this case showed that coexistence of chr 17p loss and chromothripsis in SHH medulloblastoma may indicate p53 pathway dysregulation and a "very high risk" tumor, even though the mutation status is wild-type. Our data also suggests insufficiency with TP53 mutation analysis and need to detect chromothripsis for finding p53-defficient tumors among SHH subgroup. From a clinical perspective, we should treat such cases with the most intensive treatment and continue to seek novel treatment strategies.

\section{Conflict of Interest Statement}

The authors declare that they have no conflict of interest.

\section{Reference}

1. Ramaswamy V, Remke M, Bouffet E, et al. Risk stratification of childhood medulloblastoma in the molecular era: the current consensus.Acta Neuropathol. 2016;131(6):821-831.

2. Taylor MD, Northcott PA, Korshunov A, et al. Molecular subgroups of medulloblastoma: the current consensus. Acta Neuropathol.2012;123(4):465-472.

3. Cavenee WK, Louis DN, Ohgaki H, Wiestler OD, International Agency for Research on Cancer. WHO classification of tumours of the central nervous system. Revised 4th edition. ed. Lyon: International Agency For Research On Cancer; 2016. 184 p.

4. Zhukova N, Ramaswamy V, Remke M, et al. Subgroup-specific prognostic implications of TP53 mutation in medulloblastoma. J Clin Oncol.2013;31(23):2927-2935.

5. Louis DN, Perry A, Reifenberger G, et al. The 2016 World Health Organization Classification of Tumors of the Central Nervous System: a summary. Acta Neuropathol. 2016;131(6):803-820.

6. Rausch T, Jones DT, Zapatka M, et al. Genome sequencing of pediatric medulloblastoma links catastrophic DNA rearrangements with TP53 mutations. Cell. 2012;148(1-2):59-71.

7. Capper D, Jones DTW, Sill M, et al. DNA methylation-based classification of central nervous system tumours. Nature.2018;555(7697):469-474.

8. Cavalli FMG, Remke M, Rampasek L, et al. Intertumoral Heterogeneity within Medulloblastoma Subgroups. Cancer Cell. 2017;31(6):737-754 e736.

9. Umehara T, Arita H, Yoshioka E, et al. Distribution differences in prognostic copy number alteration profiles in IDH-wild-type glioblastoma cause survival discrepancies across cohorts. Acta Neuropathol Commun. 2019;7(1):99.

10. Shih DJ, Northcott PA, Remke M, et al. Cytogenetic prognostication within medulloblastoma subgroups. J Clin Oncol.2014;32(9):886-896.

11. Liu Y, Chen C, Xu Z, et al. Deletions linked to TP53 loss drive cancer through p53-independent mechanisms. Nature.2016;531(7595):471-475.

12. Kool M, Korshunov A, Remke M, et al. Molecular subgroups of medulloblastoma: an international meta-analysis of transcriptome, genetic aberrations, and clinical data of WNT, SHH, Group 3, and Group 4 medulloblastomas. Acta Neuropathol. 2012;123(4):473-484.

13. Schwalbe EC, Lindsey JC, Nakjang S, et al. Novel molecular subgroups for clinical classification and outcome prediction in childhood medulloblastoma: a cohort study. The Lancet Oncology.2017;18(7):958-971. 
14. Korshunov A, Remke M, Kool M, et al. Biological and clinical heterogeneity of MYCN-amplified medulloblastoma. Acta Neuropathol. 2012;123(4):515-527.

\section{Figure Legends}

Figure 1.

(A, B) Sagittal and axial gadolinium (Gd)-enhanced T1-weighed image showing a posterior fossa tumor obstructing the fourth ventricle at diagnosis.

(C) Sagittal Gd-enhanced T1-weighed image of the spine at relapse presenting multiple disseminated lesions.

(D) Histopathological findings (hematoxylin and eosin). Densely packed pleomorphic undifferentiated tumor cells were observed with prominent nucleoli, cell wrapping, and apoptotic bodies.

(E) Interphase fluorescence in situ hybridization using Vysis LSI MYCN (2p24) Spectrum Green / CEP 2 (2p11.1-q11.1) Spectrum Orange Dual Color Probe shows MYCN amplification.

(F) Immunohistochemical staining for p53 was negative.

Figure 2. Copy number plotting demonstrating $M Y C N$ amplification, loss of chromosome $17 \mathrm{p}$, and chromosome 13 suggestive of chromothripsis.
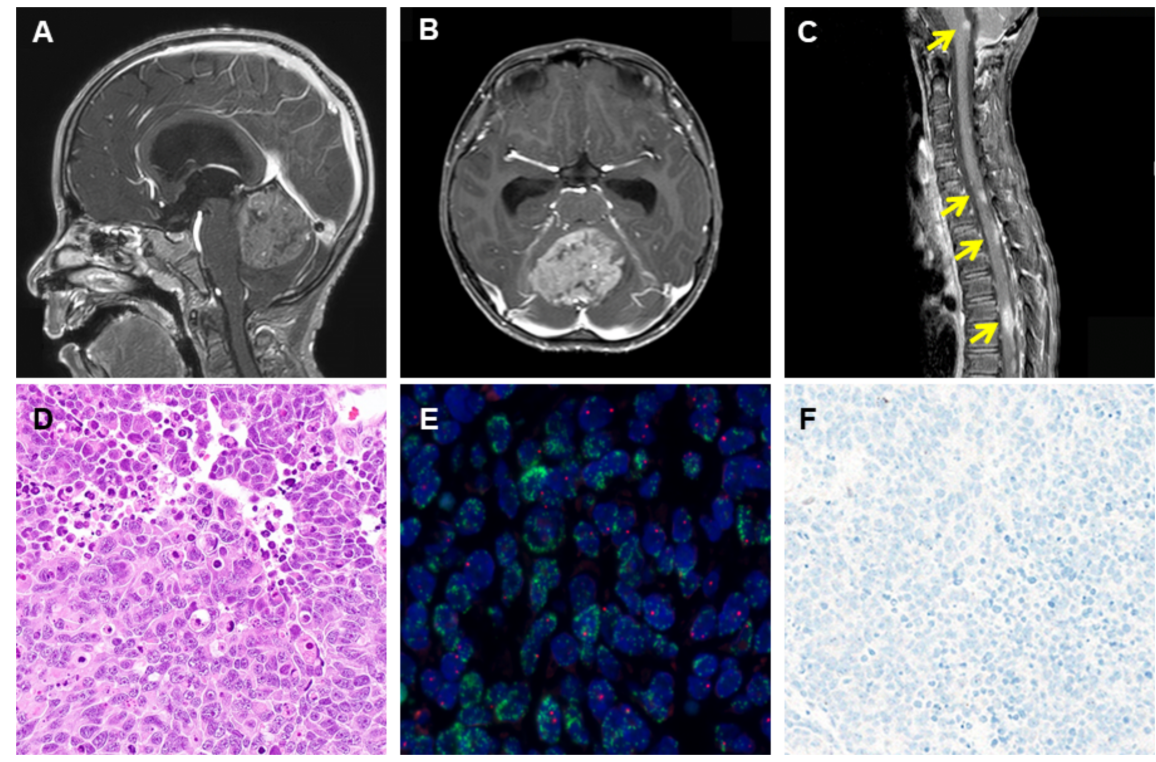

$F$ 


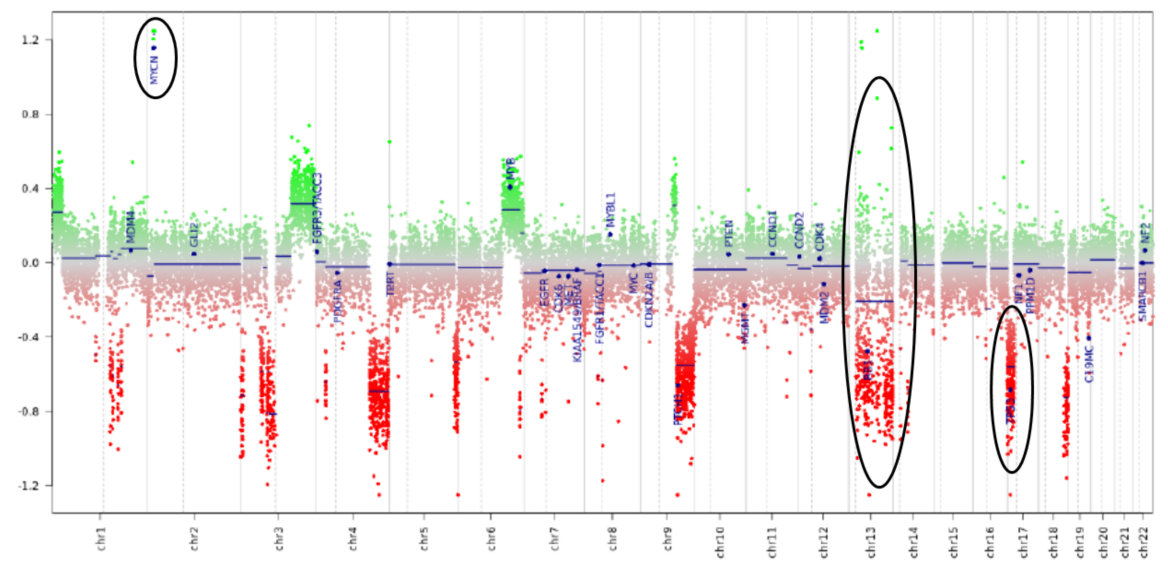

